\title{
Implementation of pharmacists' interventions and assessment of medication errors in an intensive care unit of a Chinese tertiary hospital
}

This article was published in the following Dove Press journal:

Therapeutics and Clinical Risk Management

9 October 2014

Number of times this article has been viewed

\author{
Sai-Ping Jiang ${ }^{1, *}$ \\ Jian Chen ${ }^{2, *}$ \\ Xing-Guo Zhang' \\ Xiao-Yang Lu' \\ Qing-Wei Zhao' \\ 'Department of Pharmacy, ${ }^{2}$ Intensive \\ Care Unit, the First Affiliated Hospital, \\ College of Medicine, Zhejiang \\ University, Hangzhou, People's \\ Republic of China \\ *These authors contributed equally \\ to this work
}

Background: Pharmacist interventions and medication errors potentially differ between the People's Republic of China and other countries. This study aimed to report interventions administered by clinical pharmacists and analyze medication errors in an intensive care unit (ICU) in a tertiary hospital in People's Republic of China.

Method: A prospective, noncomparative, 6-month observational study was conducted in a general ICU of a tertiary hospital in the People's Republic of China. Clinical pharmacists performed interventions to prevent or resolve medication errors during daily rounds and documented all of these interventions and medication errors. Such interventions and medication errors were categorized and then analyzed.

Results: During the 6-month observation period, a total of 489 pharmacist interventions were reported. Approximately 407 (83.2\%) pharmacist interventions were accepted by ICU physicians The incidence rate of medication errors was 124.7 per 1,000 patient-days. Improper drug frequency or dosing $(\mathrm{n}=152,37.3 \%)$, drug omission $(\mathrm{n}=83,20.4 \%)$, and potential or actual occurrence of adverse drug reaction $(n=54,13.3 \%$ ) were the three most commonly committed medication errors. Approximately 339 (83.4\%) medication errors did not pose any risks to the patients. Antimicrobials ( $n=171,35.0 \%)$ were the most frequent type of medication associated with errors.

Conclusion: Medication errors during prescription frequently occurred in an ICU of a tertiary hospital in the People's Republic of China. Pharmacist interventions were also efficient in preventing medication errors.

Keywords: pharmacist, medication error, prevalence rate, type, severity, intensive care unit

\section{Introduction}

Medication errors (MEs) are among the most common preventable causes of adverse drug events. ${ }^{1,2}$ In 21 hospitals in the Netherlands, a retrospective study showed that $>15 \%$ of adverse events were related to medications, and $21.2 \%$ of these events were considered preventable. ${ }^{3}$ In the Middle East, ME rates varied from $7.1 \%$ to $90.5 \%$ of prescription errors and from $9.4 \%$ to $80 \%$ of administration errors. ${ }^{4}$

Patients admitted in intensive care units (ICUs) are at a high risk of MEs because of critical illness, complex ICU environment, multiple medications, and frequent changes in medication therapy. ${ }^{5-8}$ Camiré et $\mathrm{al}^{9}$ reviewed data from 205 ICUs in 29 countries and found that the prevalence of MEs in ICUs was approximately 10.5 per 100 patient-days. Such a frequency of MEs was similar between prescription (54\%) and administration (46\%) phases. However, ME definitions and detection methods vary greatly in different reports. ${ }^{5}$

MEs may cause inefficient disease management for patients, additional workload for pharmacy personnel, increased cost for pharmacies and patients, frustration among
Correspondence: Qing-Wei Zhao Department of Pharmacy, the First Affiliated Hospital, College of Medicine, Zhejiang University, 79 Qingchun Road, Hangzhou, 3 I0003, People's Republic of China

Tel $+8657|872334| I$

Fax +86 57I 8723 34II

Email qingweizhao66@yeah.net 
patients and pharmacy staff, and increased patient morbidity and mortality. ${ }^{5,10}$ However, data regarding MEs in the People's Republic of China remain limited; no information related to MEs in ICUs of Chinese tertiary hospitals has also been provided. In the United States, $62.2 \%$ of ICUs are equipped with clinical pharmacy services. ${ }^{11,12}$ The presence of clinical pharmacists in ICUs has reduced the number of adverse events, improved cost savings and medications, decreased mortality rates during ICU stay, and shortened the duration of ICU admission. ${ }^{13-15}$

In the People's Republic of China, full-time clinical pharmacy services only began in 2005 after the People's Republic of China Ministry of Health issued a series of documentations regarding clinical pharmacists. Given differences in cultures, laws, politics, clinical settings, and health care systems, the implementation of pharmacy services in ICU possibly varies between the People's Republic of China and other countries. This study aimed to describe the development and implementation of full-time clinical pharmacy services in an ICU in People's Republic of China. This study also aimed to evaluate MEs encountered in the ICU of a tertiary hospital in the People's Republic of China. We also determined the parameters used to assess ME-related issues, such as prevalence rate, type, severity, and relevant medications.

\section{Methods}

\section{Setting}

The study was conducted in a general ICU of a university-affiliated tertiary hospital with 33 beds. The medical staff in the ICU comprised state-certified intensivists, ICU fellows and residents, registered nurses, respiratory therapists, a full-time critical care pharmacist, and two resident pharmacists.

\section{Study design}

A prospective, noncomparative, 6-month observational study was performed from January 1, 2013 to June 30, 2013. This study aimed to evaluate the interventions of clinical pharmacists and analyze the MEs encountered in the ICU of a tertiary hospital in the People's Republic of China. All of the patients admitted to the ICU during the study period were included. If a patient was admitted and subsequently discharged without pharmacists' participation, related medication orders were excluded in the calculation of results; no other exclusion criteria were applied. Ethical approval was acquired from the Research Review Committee of the hospital.

\section{Pharmacist interventions}

Pharmacy services were provided 8 hours a day, 5 days a week. Patient medication orders were reviewed by an experienced pharmacist and two pharmacist residents during daily clinical rounds and during pharmaceutical ward rounds. The monitored items included indications, contraindications, drug selection, drug-therapy duplication, drug dosage, drug administration, drug-treatment duration, potential drug-drug reactions, and adverse drug reaction (ADR)-related problems. Local evidence-based pharmacotherapy protocols and national and international pharmacotherapy guidelines were adopted for pharmacy reviewing.

With pharmacy services in the ICU, the interventions administered by the pharmacists were recorded in detail. For this purpose, the pharmacists completed a registration form for each intervention after they reviewed their medication orders, consulted with physicians, or administered other pharmacy services. The registration form completed by the pharmacists consists of the following items: specific date; patients' baseline characteristics (sex, age, Acute Physiology and Chronic Health Evaluation II [APACHE II] score and diagnosis); length of ICU stay; number of drugs used in ICU; specific recommendations; medication details; to whom pharmaceutical activities were initiated; and whether the recommendations were accepted by the ICU physicians. All of the interventions were then evaluated in terms of intervention type, acceptance rate, and to whom pharmaceutical activities were initiated.

\section{Assessment of MEs}

Any error found in medication prescription, transcription, preparation, dispensation, and administration is defined as a ME. ${ }^{5}$ In this study, MEs referred to prescription errors; errors related to other processes were not evaluated. Each medication order detected with a potential ME was discussed by the ICU pharmacists with the attending ICU physicians. If the attending ICU physicians agreed with the pharmacists' recommendations, then this medication order was changed and scored as an ME. If the attending ICU physicians did not accept the pharmacists' recommendations, the detected potential ME was regarded as appropriate. ${ }^{14}$ The rate of $>80 \%$ was considered well-accepted by the physicians in this study.

The prevalence rate, type, severity, and medications related to MEs were analyzed. The prevalence rate of MEs was expressed as the incidence rate of MEs per 1,000 monitored patient-days and calculated from the total number of MEs in ICU divided by the patients' total length of ICU stay and multiplied by 1,000. The severity of MEs was evaluated according to the National Coordinating Council for Medication Error Reporting and Prevention (NCC-MERP) classification; ${ }^{14,16}$ this classification was divided into four categories according to the severity of the outcome: 1) MEs 
occurred without posing harm to patients (subcategories B and C);2) MEs caused potential harm to patients (subcategory D); 3) MEs caused harm to patients (subcategories E, F, G, and H); and 4) MEs resulted in a patient's death (subcategory I).

\section{Data collection and analysis}

Data collected were encoded by a pharmacist resident into Microsoft Excel 2007 (Microsoft Corporation, Redmond, WA, USA). Variables, including patients' characteristics, type of recommendations, consensus rate, source of interventions, and ME-related concerns, such as prevalence rate, type and severity, and relevant medications, were analyzed.

\section{Results \\ Patient characteristics}

Patient characteristics are shown in Table 1. Among these patients, $403(69.1 \%)$ were male; furthermore, $153(26.2 \%)$ suffered from renal insufficiency with an estimated creatinine clearance of $\leq 50 \mathrm{~mL} / \mathrm{min}$ upon admission or during ICU stay. A total of 99 (17.0\%) were treated with renal replacement therapy (RRT) because of

Table I Patient characteristic during observational period in an ICU of a Chinese tertiary hospital

\begin{tabular}{ll}
\hline Characteristics & $\mathbf{n}(\%)$ \\
\hline $\mathrm{n}$ & $583(100)$ \\
Male & $403(69.1)$ \\
Female & $180(30.9)$ \\
Age (years) & $60.7 \pm 21.9$ \\
Weight (kg) & $62.4 \pm 25.8$ \\
APACHE Il score & $14.8 \pm 8.9$ \\
Patients with renal insufficiency* & $153(26.2)$ \\
Patients receiving renal replacement therapy & $99(17.0)$ \\
Admission diagnosis n (\%) & \\
Pneumonia and/or ARDS & $137(23.5)$ \\
CHF/pulmonary edema & $83(14.2)$ \\
Cerebrovascular accident & $73(12.5)$ \\
Cardiac/aortic surgery & $65(11.1)$ \\
Septic shock & $51(8.7)$ \\
Gastrointestinal surgery & $43(7.4)$ \\
MODS & $28(4.8)$ \\
Others & $103(17.7)$ \\
Length of ICU stay (d) & $5.6 \pm 4.5$ \\
Drugs used per admission & $6.5 \pm 4.6$ \\
\hline
\end{tabular}

Notes: Data are expressed as $\mathrm{n}(\%)$ or mean \pm standard deviation. *Patients with renal insufficiency were defined as patients older than 18 years and who had an estimated creatinine clearance less than or equal to $50 \mathrm{~mL} / \mathrm{min}$ upon admission or during the ICU stay. "Others" includes trauma, severe pancreatitis, hyperkalemia, and hypercalcemia.

Abbreviations: APACHE II, Acute Physiology and Chronic Health Evaluation II; ARDS, adult respiratory distress syndrome; CHF, congestive heart failure; ICU, intensive care unit; MODS, multiple organ dysfunction syndrome. renal failure or acute kidney injury. The most frequently diagnosed illnesses in ICU admission were pneumonia and/or adult respiratory distress syndrome $(n=137,23.5 \%)$ followed by congestive heart failure/pulmonary edema $(n=83,14.2 \%)$.

\section{Implementation of pharmacist interventions}

The pharmacists presented 489 recommendations to patients in the ICU during the observation period. Changes in drug frequency or dosage were the most frequent type of recommendations ( $\mathrm{n}=178,36.4 \%)$; among these changes, the majority (133 of 178, 74.7\%) included dosage adjustment for patients with renal insufficiency or those subjected to RRT (Table 2).

Among 489 pharmacists' recommendations, 338 (69.1\%) were proactive; that is, these recommendations were initiated by the pharmacists, and $151(30.9 \%)$ were initiated by medical/nursing staff when the pharmacists were in the ward or contacted via email/phone. Approximately 407 (83.2\%) of the pharmacists' recommendations could be accepted by the physicians (Table 2). Thus, 407 MEs were possibly prevented because of pharmacist intervention. The acceptance rate among the types of recommendations was $\geq 70 \%$. The acceptance rate of the recommendations of wrong drug selection or indication was lower (51.2\%) than that of other types of recommendations. The recommendations related to the provision of drug information or the discontinued use of drugs because of contraindication could be $100 \%$ accepted by the physicians.

\section{Assessment of MEs}

Accordingly, 407 MEs were prevented by pharmacist interventions during the observation period. As a result, the incidence rate of MEs was approximately 124.7 per 1,000 monitored patient-days. Improper drug frequency or dosing ( $\mathrm{n}=152,37.3 \%)$, drug omission $(\mathrm{n}=83,20.4 \%)$, and potential or actual occurrence of ADR $(n=54,13.3 \%)$ were the three most common MEs (Figure 1). Patients with renal insufficiency experienced MEs at a rate 2.3-times greater than those with normal renal function. The severity of MEs was further evaluated by NCC-MERP classification. As a result, 339 (83.4\%) MEs posed no harm (subcategories B and C) to the patients, $48(12.0 \%)$ were potentially harmful (subcategory D) to the patients, and 14 (3.4\%) could actually harm the patients (preventable adverse drug event, subcategories $\mathrm{E}$ and $\mathrm{F}$ ).

More than eight types of medications could cause MEs (Figure 2). Antimicrobials were the most frequent type 
Table 2 Type and number of pharmacist's recommendations in an intensive care unit of a Chinese tertiary hospital

\begin{tabular}{lll}
\hline Category of pharmacist recommendations & $\mathbf{n}(\%)$ recommendations & $\mathbf{n}(\%)$ recommendations accepted \\
\hline Change in drug frequency or dosing: improper drug frequency or dosing & $178(36.4)$ & $149(83.7)$ \\
Start new drugs: lack of use of needed drugs & $81(16.6)$ & $72(88.9)$ \\
Change or stop drugs: potential ADR or occurrence of ADR & $69(14.1)$ & $54(78.3)$ \\
Provision of drug information: lack of drug-related information & $49(10.0)$ & $49(100)$ \\
Change or stop drugs: improper drug selection or indication & $41(8.4)$ & $21(51.2)$ \\
Change or stop drugs: improper duration of drug treatment & $27(5.5)$ & $22(81.5)$ \\
Change or stop drugs: drug-drug interactions & $18(3.7)$ & $16(88.9)$ \\
Stop drugs: contraindication & $11(2.3)$ & $11(100)$ \\
Others: wrong solvent, duplication, etc. & $15(3.1)$ & $13(86.7)$ \\
Total & $489(100)$ & $407(83.2)$ \\
\hline
\end{tabular}

Abbreviation: ADR, adverse drug reaction.

of MEs ( $\mathrm{n}=171,35.0 \%)$ followed by cardiovascular drugs $(n=76,15.5 \%)$ and enteral/parenteral nutrition drugs $(n=62$, $12.7 \%)$. Considering specific drugs, we noted piperacillin/sulbactam $(n=48,9.8 \%)$, enteral nutrition drugs $(n=76$, $15.5 \%)$, and meropenem or imipenem $(n=36,7.4 \%)$ as drugs most commonly associated with MEs.

\section{Discussion}

Cultures, laws, politics, clinical settings, and health care systems differ between the People's Republic of China and other countries, such as the US or the UK. Moreover, the participation of pharmacists in ICU teams in the People's Republic of China has been recently established. However, reports on the analysis of pharmacist interventions and the measurement of MEs in ICUs in the People's Republic of China remain limited. To our knowledge, this study is the first to investigate pharmacist interventions and assess MEs in an ICU of a hospital in the People's Republic of China. This study provided insights into the value of pharmacy services in the People's Republic of China's health care system.

A high number of recommendations accepted by the physicians indicated that pharmacists in ICUs were well accepted by physicians. However, acceptance rate was greatly dependent on the type of recommendations. Extremely important recommendations (drug contraindication) or urgent need for physicians (consulting regarding drug information) could reach a high acceptance rate (100\%); by contrast, recommendations requiring specific clinical skills from pharmacists (drug selection) presented a low acceptance rate $(51.2 \%)$. Despite remarkable improvements in pharmacy services in hospitals in the People's Republic of China, clinical skills of clinical pharmacists are essential for pharmaceutical care in a patient-centered pharmacy setting. Therefore, this area should be further improved.

In this study, the incidence rate of MEs was approximately 124.7 per 1,000 monitored patient-days. This finding is close to that described by Camiré et al in which 29 countries

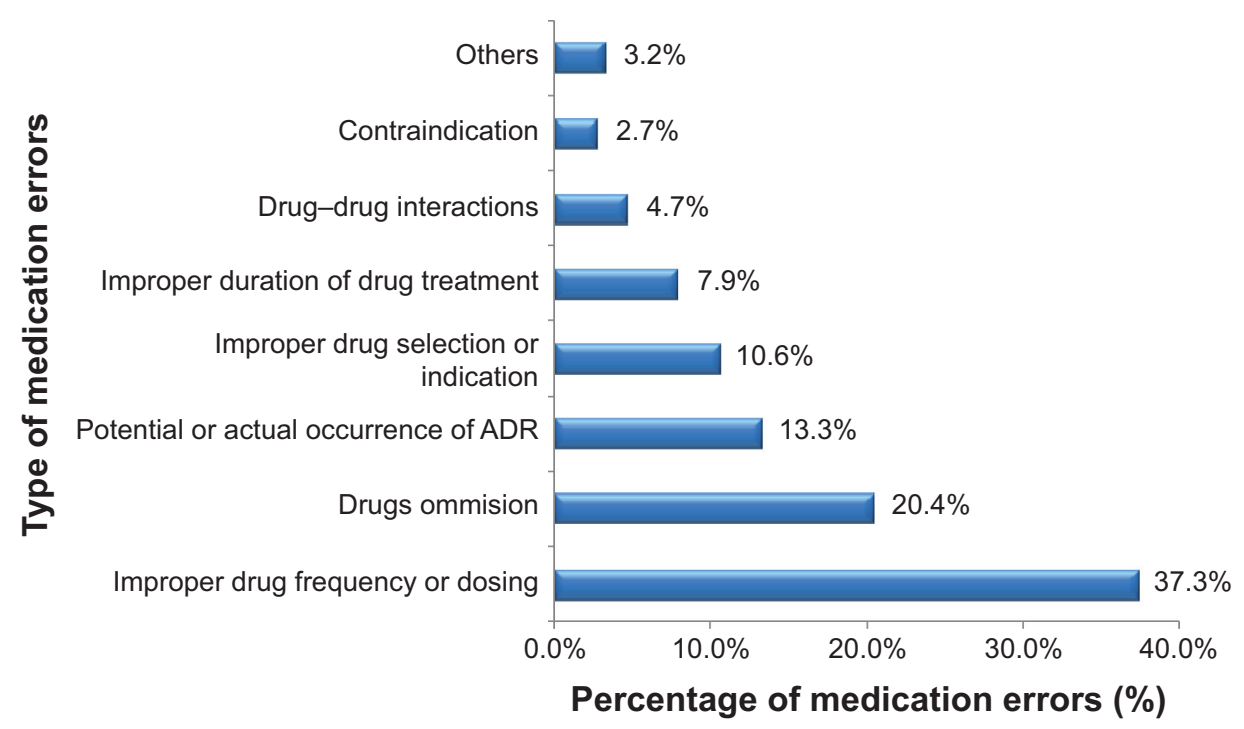

Figure I Type of medication errors encountered in an intensive care unit of a Chinese tertiary hospital.

Note: "Others" refer to medication errors, including wrong solvent, duplicated drug use, and so on.

Abbreviation: ADR, adverse drug reaction. 


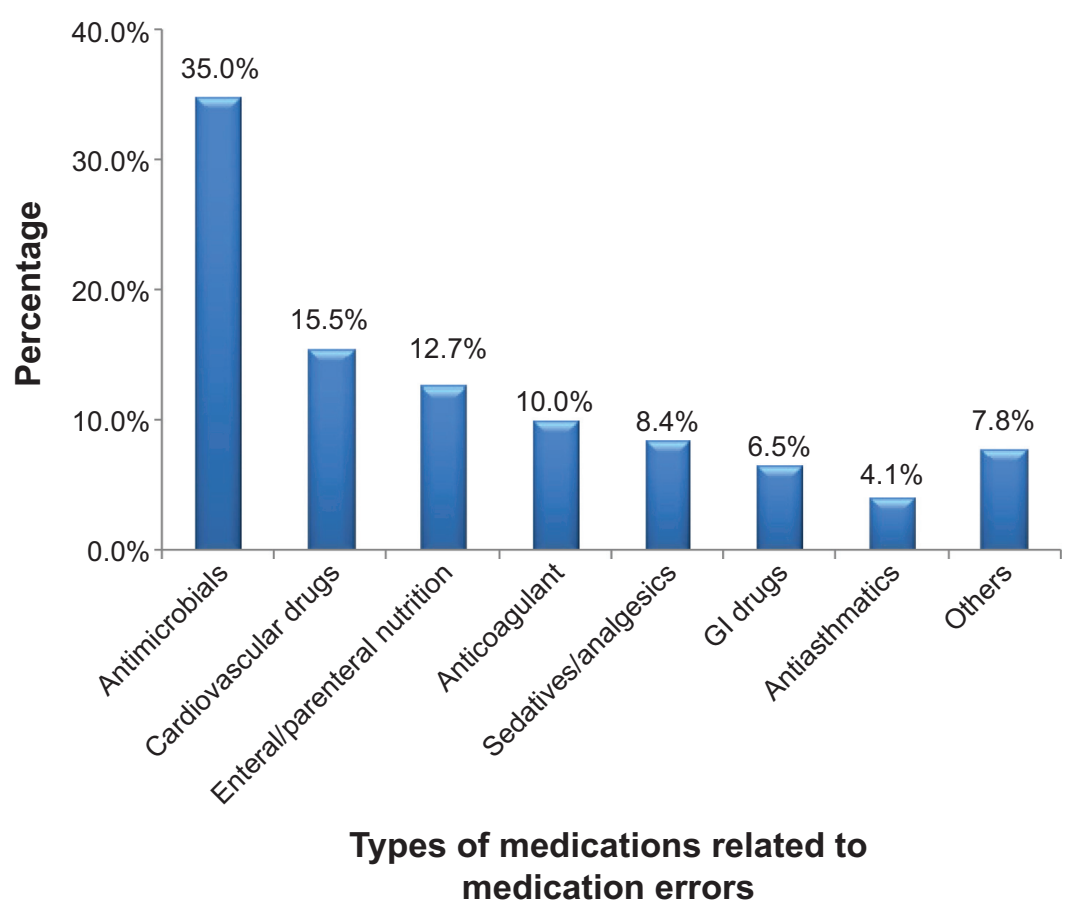

Figure 2 Type of medication with medication errors in an intensive care unit of a Chinese tertiary hospital.

Note: "Others" refer to corticosteroids, antidiabetics, electrolytes, hepatinica, and central nervous system drugs.

Abbreviation: $\mathrm{Gl}$, gastrointestinal.

with 205 ICUs are included. ${ }^{9}$ However, the prevalence rate of MEs in ICUs reported from different countries considerably varies. The following prevalence rates have been recorded: 276 per 1,000 patient-days in two ICUs in Northeastern USA ${ }^{17}$ 62.5-190.5 per 1,000 monitored patient-days in a Dutch ICU; ${ }^{14}$ and 65.1 per 1,000 patient-days in two pediatric ICUs in two tertiary-care teaching hospitals in Japan. ${ }^{18}$ These results could possibly be attributed to the differences in the ICUs of hospitals in different countries, as well as the type of ICUs, definitions of MEs, methods used to detect MEs, and other human factors (including stress, high workload, and knowledge deficits). ${ }^{19,20}$

The three most common MEs were improper drug frequency or dosage, drug omission, and potential or actual occurrence of ADR. Agalu ${ }^{19}$ reported that incorrect drug combination, drug omission, and wrong frequency are the three most common MEs in ICUs. By contrast, our findings are slightly similar to those of Klopotowska, ${ }^{14}$ in which the majority of MEs are related to drug- or dose-omission errors. Approximately $26.2 \%$ of patients in our department suffered from renal insufficiency and $16.7 \%$ of these patients received RRT. Dosage adjustment was frequently required for these patients when prescribed drugs were cleared in the renal system. The pharmacological knowledge of health care providers is an independent predictor of MEs. ${ }^{21}$ Although physicians are skilled in diagnosing diseases, they may be unfamiliar with the pharmacokinetics of prescribed medications. This condition is particularly true for younger physicians, such as physician residents and fellows, who are possibly at a high risk of committing dosage errors. The involvement of a pharmacist at the bedside with a medical team may help physicians understand the prescribed medications.

Antimicrobials, cardiovascular drugs, and enteral/ parenteral nutrition, which are frequently prescribed or changed by ICU physicians, are at a higher risk of MEs than other medications. These findings are similar to those of Agalu et al, and Klopotowska et al, in which antibiotics are the most common drugs subjected to prescription errors. ${ }^{14,19}$ This result is possibly because antibiotics are the most-frequently prescribed and/or changed drugs in ICUs. However, the results from an ICU in Pennsylvania, USA were quite different; in particular, opioid analgesics, betalactam antimicrobials, and blood coagulation modifiers were the three most common drugs associated with MEs in the Pennsylvania study. ${ }^{22}$ This difference may be due to different types of patients in various ICUs.

Many strategies have been proposed to prevent MEs. These include optimizing medication processes, eliminating situational risk factors, adequate staffing, and promoting pharmacist participation in ICU care. ${ }^{5}$ The influence of clinical pharmacists in ICUs has reduced the number of adverse effects, decreased mortality rates during ICU stay, improved medication dosage and cost savings, and shortened the duration of ICU stay. ${ }^{13-15}$ Whether a pharmacist providing critical 
care based on the Chinese health care system reduces adverse drug events, enhances cost savings, or provides other values will be investigated in our future studies.

Therefore, this study demonstrated that the presence of pharmacists in the ICU was well-accepted by medical teams in the People's Republic of China. A total of 407 MEs were possibly prevented by pharmacist interventions. The incidence rate of MEs was approximately 124.7 per 1,000 patient-days. The assessment of MEs revealed that improper drug frequency or dosage, drug omission, and potential or actual occurrence of ADR were the three most common types of MEs, and $83.4 \%$ of MEs did not pose any risk to patients. Antimicrobials were most commonly associated with MEs. These results will help understand MEs commonly encountered in an ICU in the People's Republic of China and enhance our pharmacy services in the future.

\section{Acknowledgment}

This work was sponsored by the National Construction Project of Key Clinical Specialties.

\section{Disclosure}

The authors report no conflicts of interest in this work.

\section{References}

1. Mansouri A, Ahmadvand A, Hadjibabaie M, et al. A review of medication errors in iran: sources, underreporting reasons and preventive measures. Iran J Pharm Res. 2014;13(1):3-17.

2. Saedder EA, Brock B, Nielsen LP, Bonnerup DK, Lisby M. Identifying high-risk medication: a systematic literature review. Eur J Clin Pharmacol. 2014;70(6):637-645.

3. Zegers M, de Bruijne MC, Wagner C, et al. Adverse events and potentially preventable deaths in Dutch hospitals: results of a retrospective patient record review study. Qual Saf Health Care. 2009;18:297-302.

4. Alsulami Z, Conroy S, Choonara I. Medication errors in the Middle East countries: a systematic review of the literature. Eur J Clin Pharmacol. 2013;69(4):995-1008.

5. Moyen E, Camiré E, Stelfox HT. Clinical review: medication errors in critical care. Crit Care. 2008;12(2):208.

6. Anthes AM, Harinstein LM, Smithburger PL, Seybert AL, Kane-Gill SL. Improving adverse drug event detection in critically ill patients through screening intensive care unit transfer summaries. Pharmacoepidemiol Drug Saf. 2013;22(5):510-516.
7. Kopp BJ, Erstadt BL, Allen ME, Theodorou AA, Priestley G. Medication errors and adverse drug events in an intensive care unit: direct observation approach for detection. Crit Care Med. 2006;34:415-425.

8. Louie K, Wilmer A, Wong H, Grubisic M, Ayas N, Dodek P. Medication error reporting systems: a survey of canadian intensive care units. Can J Hosp Pharm. 2010;63(1):20-24.

9. Camiré E, Moyne E, Stelfox HT. Medication errors in critical care: risk factors, prevention and disclosure. CMAJ. 2009;180(9):936-943.

10. Odukoya OK, Stone JA, Chui MA. E-prescribing errors in community pharmacies: exploring consequences and contributing factors. Int J Med Inform. 2014;83(6):427-437.

11. Maclaren R, Devlin JW, Martin SJ, Dasta JF, Rudis MI, Bond CA. Critical care pharmacy services in United States hospitals. Ann Pharmacother. 2006;40(4):612-618.

12. Jiang SP, Zhu ZY, Wu XL, Lu XY, Zhang XG, Wu BH. Effectiveness of pharmacist dosing adjustment for critically ill patients receiving continuous renal replacement therapy: a comparative study. Ther Clin Risk Manag. 2014;10:405-412.

13. Ernst AA, Weiss SJ, Sullivan A 4th, et al. On-site pharmacists in the ED improve medical errors. Am J Emerg Med. 2012;30(5):717-725.

14. Klopotowska JE, Kuiper R, van Kan HJ, et al. On-ward participation of a hospital pharmacist in a Dutch intensive care unit reduces prescribing errors and related patient harm: an intervention study. Crit Care. 2010;14:R174.

15. MacLaren R, Bond CA, Martin SJ, Fike D. Clinical and economic outcomes of involving pharmacists in the direct care of critically ill patients with infections. Crit Care Med. 2008;36(12):3184-3189.

16. http://www.nccmerp.org [homepage on the Internet]. Taxonomy of Medication Errors. The National Coordinating Council for Medication Error Reporting and Prevention; 2014. Available from: http://www. nccmerp.org. Accessed.

17. Carayon P, Wetterneck TB, Cartmill R, et al. Characterising the complexity of medication safety using a human factors approach: an observational study in two intensive care units. BMJ Qual Saf. 2014;23(1):56-65.

18. Sakuma M, Ida H, Nakamura T, et al. Adverse drug events and medication errors in Japanese paediatric inpatients: a retrospective cohort study. BMJ Qual Saf. Epub 2014 Apr 17.

19. Agalu A, Ayele Y, Bedada W, Woldie M. Medication prescribing errors in the intensive care unit of Jimma University Specialized Hospital, Southwest Ethiopia. J Multidiscip Healthc. 2011;4:377-382.

20. Frith KH. Medication errors in the intensive care unit: literature review using the SEIPS model. AACN Adv Crit Care. 2013;24(4):389-404.

21. Krähenbühl-Melcher A, Schlienger R, Lampert M, Haschke M, Drewe J, Krähenbühl S. Drug-related problems in hospitals: a review of the recent literature. Drug Saf. 2007;30:379-407.

22. Kane-Gill SL, Kowiatek JG, Weber RJ. A comparison of voluntarily reported medication errors in intensive care and general care units. Qual Saf Health Care. 2010;19(1):55-59.
Therapeutics and Clinical Risk Management

\section{Publish your work in this journal}

Therapeutics and Clinical Risk Management is an international, peerreviewed journal of clinical therapeutics and risk management, focusing on concise rapid reporting of clinical studies in all therapeutic areas, outcomes, safety, and programs for the effective, safe, and sustained use of medicines. This journal is indexed on PubMed Central, CAS,

\section{Dovepress}

EMBase, Scopus and the Elsevier Bibliographic databases. The manuscript management system is completely online and includes a very quick and fair peer-review system, which is all easy to use. Visit $\mathrm{http}: / /$ www.dovepress.com/testimonials.php to read real quotes from published authors. 